ISAHP 1996, Vancouver, Canadh, July 12-15, 1996

\title{
A MULTI-CRITERIA COMPARISON OF RESPONSE SCALES AND SCALING METHODS IN THE AHP ${ }^{1}$
}

\author{
David V. Budescu, Bradley D. Crouch and Osvaldo F. Morera \\ Department ofP sydhology \\ The University of 1 limois a Urtana-Champaign \\ dbudescr@s.psych.uiuc.edu
}

\begin{abstract}
Two methodological byproducts of the recent intensive work within the Analytical Hierarchy Process (AHP) framework are (1) the devdopment of new (and refinement of some old) scaling techniques for the extraction of the priarity weights from a matrix of direct ratio judgments and (2) the derivation of altenative scales for the pairwise comparisons of the AHP. This paper compares a number of these scales in combination with some of the methods used to extract priority weights for three distinct types of pairwise comparisons in the AHP. The three types of matrices consist of between-attributes comparisons, within-attribute comparisons and global comparisons (Jensen, 1984a). The methods and scales are compared in terms of several criteria inchuding the solution's goodness of fit, the variance and entropy of the pricrity weights and the number of order violations. Results indicate that the nature of the scale has systematic and significant effects of various chancteristics of the solution, under all scaling methods. In particular, increasing the spacing of the scale points tends to increase the difterentiation among the priarity weights, but has a negative effect on the solution's goodness of fit.

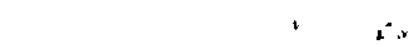

Introduction
\end{abstract}

The Analytic Hierarchy Process (AHP) is a popular multi-criteria decision methodology which was developed and disaussed extensively by Saaty (e.g 1977, 1980, 1986:1990a). Since its introduction, the AHP has been applied in a wide variety of domains and disciplines (see Zahedi, 1986a; Saaty \& Vargas, 1982, 1991 for representative lists). A typical AHP analysis consists of four interrelated stages:

(1) The decision problem is structured as a dominance hiearchy.

(2) Data are collected through a process of pairwise comparisons among ail elements at a specific levd of the hiearchy with respect to singe, well-defined, criteria from higher levels of the hieriachy.

(3) Priority weights are extracted fom each set of comparisons obtained at stage (2) through an appropriate scaling/estimation procedure.

(4) The various weighits derived at stage (3) are combined; using a particular aggregation mode, to yield an overall weight for eachalternative.

Consider, for example, a student who is looking for a campus apartment and has to choose among one of $n=5$ altenatives. At the top levd of the hienrchy lies the goal of "Selocting the best apartment". In structuring the problem, the student decides to focus on $p=5$ sub-criteria at the second levd (e.g cost, size, location, reputation of the landlord and amenities). This hiearchy is displayed Figure 1.

\footnotetext{
'Bradley D. Crouch's work was supported by a National Science Foundation Graduate Fellowship.
} 


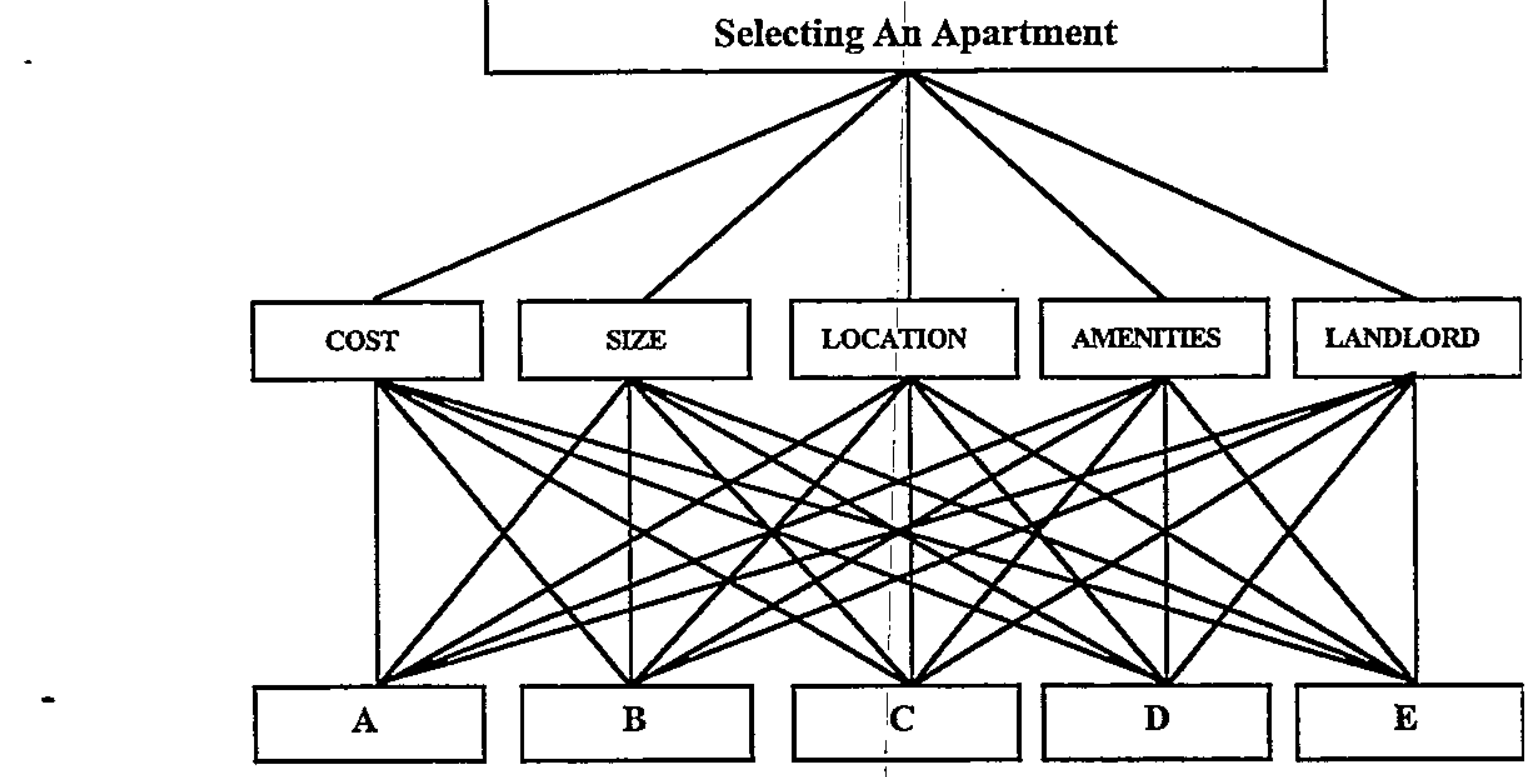

FIGURE 1. A hierarchy for apartment selection

At the second stage of the process the student compares, in pairwise fishion, all attributes (we will refir to these comparisons as between-attributes) as well as all apartments on each of the attributes (we label these withinattribute comparisons). At the third stage, each of these sets of comparisons is analyzed and priarity weights are extracted. Let $w_{i}$ be the weight assigned to the $i_{t b}$ criterion $(i=1 . p)$ at leve 2 , and $v_{i j}$ be the estimated weight of the $j_{\text {th }}$ aparment $(j=1 . . n)$ on criterion $i$. Finally, these estimates are combined to yield $V_{j}$, the infred overall weight of apartment $j$. This is defined as a simple weighted sum of the criterion specific priorities (over all criteria), i.e.:

(1)

$$
V_{j}=\sum_{i=1}^{p} w_{i} v_{i j}
$$

A simplified approach to this decision problem (Jensen, 1984a) requires the Decision Make (DM) to compare all the apartments in a pairwise fashion, considering all the criteria simultaneously (we will refr to these comparisons as global). In other words, one level of the typical AHP hierarchy is eliminated. Figure 2 depicts this simplified version of the AHP for the same decision.

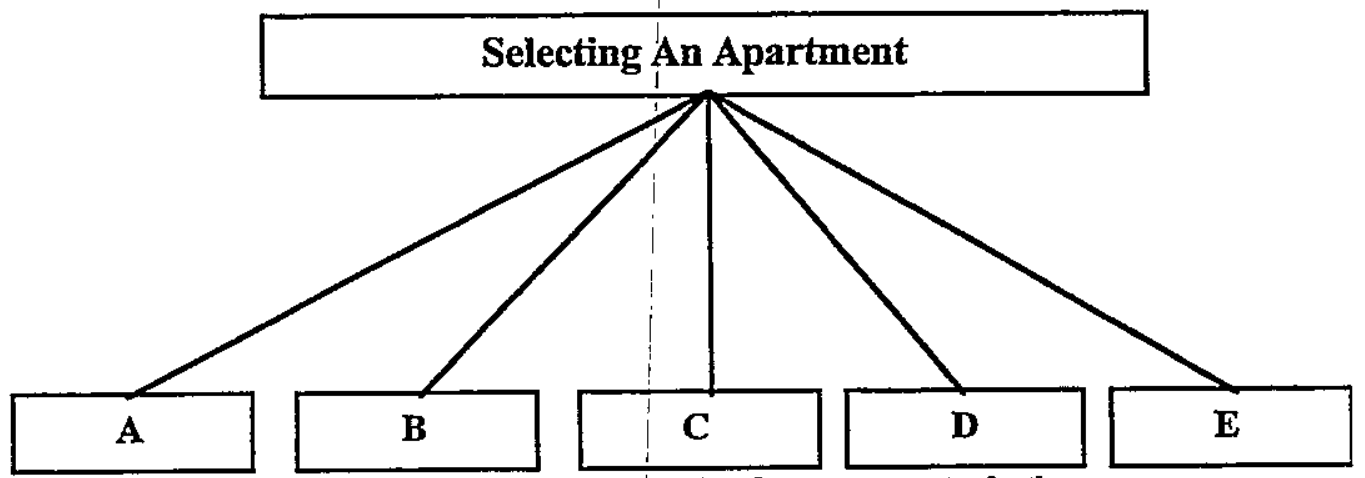

FIGURE 2. A global hierarchy for apartment selection

Two methodological byproducts of the work with AHP are: (1) The development of new (and refinement of some old) scaling and estimation techniques to be used in stage 3 of the AHP in extracting priority weights from a matrix of direct ratio judgments, and (2) The derivation of alternative scales to be used in the process of pairwise comparisons (within- and between- attributes) in the AHP. This pape provides a comparison of a number of these scales in combination with the most popular methods used to extract priority weights, by applying all scales and solutions to a set of judgments obtained as part of decision making study, in a 
meaningfil context. To motivate this work, we point out that the choices made with regard to the nature of the specific scale used in the elicitation of judgments and the scaling methodology can afect the nature of the eventual decisions (see Lootsma, 1993; Olson, Fliedner \& Curie, 1995 for recent examples).

\section{Scaling a matrix of ratio judgments: An Overview}

The ratio judgments can be represented in a square matrix $\mathbf{R}$ (with elements $r_{i j}$ ), of order $n$, with the following properties (for all $i, j=1 . . n$ ):

$$
\text { (i) Positivity: } r_{i j}>0 \text { (ii) Reciprocity: } r_{i j}=1 / r_{j i}, \quad \text { (iii) Reflexivity: } r_{i i}=1 \text {. }
$$

Assume now a simple modd relaing these overt judgments to an unobservable vector of values, $\mathbf{w}=\left(w_{1}, . . w_{n}\right)$, which represent the priority weights of the $n$ stimuli:

$$
r_{i j}=w_{i} / w_{j}
$$

Under this mode, the matrix $\mathbf{R}$ is fully detemined by the vector $\mathbf{w}$. Thus, $\mathbf{R}$ is of unit rank and, for any triple of distinct stimuli $(i, j, k=1 \ldots n)$, we expect:

$$
\text { (iv) Consistency: } r_{i j} \bullet r_{j k}=r_{i k}
$$

It follows that $R$ has a single positive eigenvalue, $\lambda=n$, and $n$-1 zero eigenvalues (Saty, 1977, 1986).

The estimation problem is to infer the set of weights, $\mathbf{w}$, underlying a given matix of empirical judgnents, $\mathbf{R}$. Under this model any column of $\mathbf{R}$ is a solution to this algebraic problem. Solutions are unique up to multiplication by a positive constant, so it is customary to normalize the weights by imposing the constraint $\Sigma w_{i}=1$. Recall however, that $\mathbf{R}$ is obtained from a sequence of fillible huma judgments and, therefore, conditions (i)-(iv) may not be satisfied. Positivity is enforced by the nature of the scale used. Typically, judgments are elicited for $n(n-1) / 2$ paiss of distinct stimuli and the rest of the entries are calaulated such that conditions (ii)-(iii) are satisfied. However, this does not guarantee consistency. In such cases, the researcher is faced with the statistical problem of estimating the weights from a redundant (and possibly, inconsistent) set of judgnents (Weber \& Borcherding 1993). Much of the work in this area has been devoted to the comparison of various estimation methods and identification of "the best" among them Partial reviews of the methods and some of their properties are found in Budescu (1984), Cook \& Kress (1992), Golany.\& Kress, (1993) and Saaty \& Vargas (1984).

Saaty $(1977,1980)$ suggested estimating $\mathbf{w}$ by $\mathbf{w}^{(t)}$, the right eigenvector of $\mathbf{R}$ corresponding to its largest eigenvalue, i.e. by solving the eigenvalue problem $\mathbf{R} \mathbf{w}^{(t)}=\lambda \mathbf{w}^{(t)}$. He also provided a simple numetical algorithm for solving this problem (we re to this as the EV solution). The inconsistency of the solution is measured by the index:

$$
\mu=(\lambda-n) /(n-1)
$$

which vanishes for perfectly consistent matrices. Saaty and Vargas (1984) present a loss finction which is optimized by this solution. Interestingly, Gulliksen $(1959,1975)$ applied a very similar procedure for ratio scaling. Cogger and Yu (1985) proposed a modification to the EV method, which is easier to compute but lacks the intuitive appeal and the optimal properties of the EV.

Jensen (1984b) descibed a Direct Least Squares (DLS) procedure, but it is seldom used because it yields multiple solutions and the computation is diffcult (Golany \& Kress, 1993). However, Chu, Kalaba \& Spingarn (1979) proposed a unique and computationally asible Weighted Least Squares (WLS) solution, w (w), which minimizes the quantity:

$$
S^{(w)}=\Sigma \Sigma\left(r_{i j} w_{i}^{(w)}-w_{j}^{(w)}\right)^{2}
$$


Barzilai, Cook \& Golany (1987), Crawford \& Williams (1985), Crawford (1987), deJang (1984) and Lootsma (1993) fiva the Logaithmic Least Squares (LLS) method. The solution $w^{(8)}$, which minimizes the quartity:

$$
S^{(g)}=\Sigma \Sigma\left[\ln \left(r_{i j}\right)-\left[\ln \left(w_{i}^{(z)} / w^{(z)}\right)\right]^{2},\right.
$$

can be shown to consist, simply, of the geometric means of the rows of $\mathbf{R}$ (it appears that Torgerson, 1958, was the first to establish this result). Due its simplicity, Saaty and Keams (1985) suggest using geometric means as an approximation to the EV solution. Because of its intuitive appeal and the ease of its computation, $\mathbf{w}^{(8)}$ has become the most popular method, next to $\mathrm{w}^{(\mathrm{r})}$.

Finally, Cook and Kress (1982) of a compelling axionatization of this problem that favors a Logaithmic Least Absolute Values (LLAV) solution. Unfartunatey, LLAV has multiple (and diffcult to calarlate) solutions.

Comparisons of the various methods (e.g Budescu, Zwi'k \& Rapoport, 1986; Golany \& Kress, 1993; Saaty 1990b; Saaty \& Vargas, 1984; Takeda, Cogger \& Yu, 1987; 1984; Zahedi, 19866) found that in most cases there is good agreement between the various solutions, and neither is unifmly superior. Whenever discrepancies between the solutions emerge, they reflect the interaction between the different loss finctions employed and specific fatures of the data For the purpose of this paper, we will focus on the three fillowing solutions, yielding unique solutions: EV, WLS and LLS solutions (denoted $\mathbf{w}^{(\mathrm{r})}, \mathbf{w}^{(w)}$, and $\mathbf{w}^{(8)}$, respectively).

\section{Ratio judgments and the nature of the scale}

Of course, all the methods described above apply to any matrix $\mathbf{R}$ satisfying requirements (i)-(iii). However, Saaty $(1977,1980)$ proposed, for theoretical and practical reasons, to restrict the ratio judgnents to the integers 1-9 and therr reciprocals (Donegan, Dodd and McMaster (1992) refer to these 17 values as "the Saaty set"). To facilitate their use, Saaty suggested using specific verbal labels in conjunction with some of these values. These labds are supposed to convey the relative impartance of the superior element within each pait, relative to the second element, and are to be applied for all comparisons. In his original paper, Saaty (1977) proposed the labeds 1: equal, 3: weak 5: essential (or strong), 7: demonstrated, 9: absolute , but others have used slightly different labes (see Pöyhönen, Hämäläinen \& Salo! 1996 and Donegan, Dodd \& McMater, 1992 fr examples).

In recent years, researchers have tried to determine whether Saaty's set is appropriate in terms of its size and the spacing of its values for all comparisons and a number of altenative scales that have been proposed. The earliest allusion to this possibility is due to Harker (1987), who showed that the eigenvalue method can be extended to the case where the observed judgments are power transforms of the Saaty set. He hinted at the possibility of employing various non-inear scales but stopped short of endorsing a specific scale, or proposing a general choice criterion.

Lootsma (1993) proposed a scale based on the assumption that human judgments follow a geometric progession with a fixed factor. The scale values in this system (called Ratio Estimation in Magnitudes or deci-Bels to Rate Altenatives which areNon-Dominated, or REMBRANDT for short) can be expressed as:

$$
\begin{array}{cc}
r^{(l)}=\exp \left[\tau\left(r_{i j}-1\right)\right] & \text { if } r_{i j} \geq 1, \\
r^{(l)}=\exp \left[-\tau\left(r_{i j}-1\right)\right] & \text { if } r_{i j}<1
\end{array}
$$

where $r_{i j}$ are the values in the Saaty set and $\tau$ is the progression factor. Lootsma recommends $\tau_{t}=\ln (2) \approx 0.7$ and $\tau_{l}=\ln (\sqrt{2}) \approx 0.35$ as "natural" factors for alternatives and criteria respectively. The most obvious problem associated with the use of a (relatively) small and restricted set of values is the inherent inconsistency with the mathematical form, and the implications of the model (Eqs. 3 and 4). For example, of the 969 distinct triples that can be generated within the Saaty set, only $45(4.6 \%)$ satisfy Eq. 4 . If all indiference judgments, i.e. $r_{i j}=1$,

${ }^{2}$ The even numbers (used to define intermediate levels) are typically not labeled. 
are eliminated, this number is further reduced to $20(2.1 \%)$ ! It fllows that a filly consistent judge would experience serious diffculties in expressing his pricrities. If for example, he thinks that both $r_{i j}$ and $r_{j k}=8$, we would expect him to judge option $i$ to be 64 times better than $k$, but this calls for values outside the restricted set. As a partial remedy for this problem, Donegan et al. (1992) and Dodd, Donegan \& McMaster (1995) proposed a Modified AHP (MAHP) in whidh the response scale is fairly linear in the middle of the scale and distinctly non-linear near the end points. Of the various possible streching finctions they selected, quite arbitrarily, $\tanh ^{-1}$, the inverse hypebolic tangent. The new scale is defined by:

$$
r^{(2)}{ }_{i j}=\exp \left\{\tanh ^{-1}\left[\left(r_{i j}-1\right) /(H-1)\right]\right\} \quad \text { if } r_{i j} \geq 1
$$

(8b)

$$
r_{i j}^{(2)}=\exp \left\{-\tanh ^{1}\left[\left(r_{i j}^{-1}-1\right) /(H-1)\right]\right\} \quad \text { if } r_{i j}<1,
$$

where $H$ is the "Horizon" parameter. An horizon of 8 , for example, yields $H=(1+14 \sqrt{3}) \approx 9.083$. This choice guarantees that the most extreme ratio (corresponding to $r_{i k}=9$ ) is obtained as a product of two ratios conesponding to $r_{i j}=r_{j k}=8$, thus solving the problem described above. Altemativey, an horizon of 7 implies, $H=(1+6 \sqrt{2}) \approx 9.485$, guaranteeing that the most extreme ratio (corresponding to $r_{i k}=9$ ) is obtained as a product of two ratios comesponding to $r_{i j}=r_{j k}=7$.

Table 1 displays values prescribed by the various scales described here for the 1-9 range. To illustrate the differences between the scales, we also list three informative statistics for each of them: The variance of the scale values, $V\left(s_{i}\right)$; the inte-quartile range of the values, $I Q R\left(s_{i}\right)$; and the variance of the "gaps" (the distances between adjacent scale values), $V\left(s_{i}-s_{i-1}\right)$. The latter is an index of the departure from linearity of the scale (note that it is 0 for Saaty's linear scale).

TABLE 1. Seven alternative nine-point scales for the $A \bar{A} \bar{P}$ and

\begin{tabular}{|c|c|c|c|c|c|c|}
\hline \multirow{2}{*}{$\begin{array}{c}\text { Saaty } \\
\alpha= \\
1.0 \\
\end{array}$} & \multicolumn{2}{|c|}{ Power } & \multicolumn{2}{|c|}{ REMBRANDT } & \multicolumn{2}{|c|}{ MAHP } \\
\hline & $\alpha=0.5$ & $\alpha=\mathbf{2 0}$ & $\tau \approx 0.7$ & $\tau=\mathbf{0 . 3 5}$ & 7-Based & 8-Based \\
\hline 1 & 1.00 & 1 & 1 & 1.00 & 1.00 & 1.00 \\
\hline 2 & 1.41 & 4 & 2 & 1.41 & 1.13 & 1.13 \\
\hline 3 & 1.73 & 9 & 4 & 2.00 & 1.27 & 1.29 \\
\hline 4 & 2.00 & 16 & 8 & 2.83 & 1.45 & 1.47 \\
\hline 5 & 2.24 & 25 & 16 & 4.00 & 1.67 & 1.72 \\
\hline 6 & 2.45 & 36 & 32 & 5.66 & 1.97 & 2.06 \\
\hline 7 & 2.65 & 49 & 64 & 8.00 & 2.42 & 2.60 \\
\hline 8 & 2.83 & 64 & 128 & 11.31 & 3.23 & 3.73 \\
\hline 9 & 3.00 & 81 & 256 & 16.00 & 5.83 & 13.93 \\
\hline \multicolumn{7}{|c|}{ Variance of scale values: $V\left(s_{i}\right)=\left[\sum s_{i}^{2}-\left(\sum s_{i}\right)^{2} / n\right] /(n-1)$} \\
\hline 7.5 & 0.45 & 788 & 7,296 & 26.00 & 3.33 & 16.88 \\
\hline \multicolumn{7}{|c|}{ Interquartile range of scale values: $I Q R\left(s_{i}\right)$} \\
\hline 4 & 0.92 & 40 & 60 & 6.00 & 1.15 & 1.31 \\
\hline \multicolumn{7}{|c|}{ Variance of gaps: $V\left(s_{i}-s_{i-I}\right)$} \\
\hline 0.0 & 0.01 & 24 & 1,960 & 2.23 & 0.70 & 12.14 \\
\hline
\end{tabular}
some summary statistics 
Each of these scales is a continuous and monotonic trans frmation of the Saaty set, and each has some desirable properties. However, they all invoke, more or less, arbitrary assumptions regading the finctional form of the key transformation and/or its parameters. Clealy, neither is unifrmly superior to the others on normative grounds. Also, in the absence of comprehensive comparative studies of the various scales (see Olsan, et al., 1995 for a recent exception) it is impossible to determine whid scale fits better most cases in an empirical sense.

\section{An empirical multi-criteria comparison of various response scales}

Previous empirical work has focused primarily on comparisons between scaling methods (EV, WLS, LLS, etc.) and has devoted only limited attention to the comparison of the various scales. In particular, there are no studies comparing systematicaly the effets of using different scales (MAFP, REMBRANDT, Power, etc.) in combination with the various solution concepts. Our study will address this issue. We will apply three scaling methods (EV, LLS and WLS) to analyze a set of matrices represented in seven distinct alternative scales (SaAy's $1-9$ scale, power transformations with $\alpha \stackrel{t}{=} 0.5$ and 2, REMBRANDT with $\tau=0.35$ and 0.7 , and MAHP with a horizon of 7 and 8 ).

The matrices consists of actual judgnents obtained from judges in real decision contexts. We will analyze three types of matrices consisting of between-attributes, within-attribute and global comparisons, in the same domain. This is an intriguing comparison. From a mathematical point of view, the various matrices are identical, but in practice DMs may trea them diffently. There are at least two justifications for this spearlation The first is "structural": Typically, the DM has more control over the selection of the relevant attributes than over the selection of the altenatives. This would suggest more homogeneity among attributes than altenatives. This appears to be the implicit assumption undelying Lootsma's (1993) recommendation to use different scaling factors fir the within-attribute and between-attribute judgnents. The second justification is "cognitive". It is conceivable that DMs invoke different psychological processes when comparing concrete alternatives versis more abstract attributes (Payne, Bettman \& Johnon, 1993).

Whenever several solutions to the same problem are compared the question of the most appropriate criterion arises. The problem is even more acute in this case when the solutions being compared vary along two dimensions. As mentioned in the introduction, various methods were designed to optimize different criteria. Obviously, none of the solution specific criteria, such as Saaty's $\mu$, can be used to compare all the solutions, although these indices are meaningfill in the context of a specific method. For example, it is informative to compare the $S^{(\mathcal{z})}$ of the various LLS solutions, but it makes littie sense to calaulate this measure for the EV or LLS solution.

- A natural and compelling criterion of comparison is the solution's external validity, i.e. its ability to capture and reproduce accurately the DM's "true" priorities. This compelling universal criterion requires direct access to one's prefrences Unfortunately, this information is rardy available, so most comparisons rely on altenative (proxy) criteria Golany \& Kress (1993) pointed out, and illustrated, the impartance of considering multiple criteria in order to obtain a complete and comprehensive understanding of the properties of the various solutions.

In the current study we will adopt a similar approach, and will compare the alternative solutions and scales along the fllowing four criteria:

(1) Thevariance of the derived weights:

$$
S^{2}\left(w_{i}\right)=\left[\sum w_{i}^{2}-1 / n\right] /(n-1) .
$$

(2) The relative entropy of the weights (Noble \& Sanchez, 1993):

$$
R E\left(w_{i}\right)=1-\sum w_{i} \ln \left(w_{i}\right) / \ln (n) .
$$

Note that $0 \leq R E \leq 1$ such that $R E=0$ when all the weights are equal, and $R E$ approaches I as one weight approaches 1 , while the other $n-1$ vanish.

The number of order reversals: The number of cases in which one altenative is judged superior to another, but assigned a lower weight by the solution. We distinguish between two types of reversals: 
(3) Strong Order Reversals (SOR) is a count of all cases in which $r_{i k}<r_{j k}$ but $w_{j}<w_{i}$, or $r_{i k}>r_{j k}$ but $w_{j}$ $>w_{i}$.

(4) Weak Order Revarsals (WOR) is a count of all cases in whidh $r_{i k} \neq r_{j k}$ but $w_{j}=w_{i}$ or $r_{i k}=r_{j k}$ but $w_{j}$ $\neq w_{i}$.

Golany \& Kress (1993) analyzed a global index of ordimal consistency, TOR, which is equivalent to (SOR + WOR / 2).

One would expect a "good" solution to be highly infarmative, differentiate well between the $n$ entities, and to have very fw order revarsals (ifany).

\section{Method}

Subjects: The present study involved 29 students from an introductory psychology class at the University of Ilinois at Urbana-Champaign that completed the study in partial fulfflment of the course requirements.

Procedure: All subjects were asked to evaluate five hypothetical apartments that varied with respect to five attributes: rent, size proximity to campus, amenities and landlord reputation. The information concerning the apartments was presented to subjects in a summary packet. All comparisons were made on a ninepoint scale.

The stimuli were constructed such that no one apartment clealy dominated any of the other four In other words, each apartment was the best in the set on one attribute, second in the set on another attribute, third on oneattribute, and so on. The apartments were constructed in this manner so that no one apartment could be obviously considered to be the "best" apatment in the global comparisons.

Fiffeen subjects assessed apartments using the global version of the AHP (Jensen, 1983), and 14 subjects used the regular (decomposed) procedure. In the latter group the between-attributes comparisons preceded the five. within-attribute sets of judgments. Experimentd sessions lasted no more than one hour

\section{Results}

The results for each of the criteria considered were analyzed and summarized in the framework of a multifactor Analysis of Variance (ANOVA) with the following three factors:

(li) Scale type Saaty's 1-9, MAHP with a horizon of 7 and 8 , REMBRANDT with $\tau_{1}=\ln (2) \approx 0.7$ and $\tau_{2}=\ln \sqrt{2}=0.35$ and power transformations with $\alpha=0.5$ and $\alpha=2.0$ (Ofcourse, Saaty's linear scale is also a member of this family of transformations with $\alpha=1$ ).

(2) Solution method: EV, LLS and WLS.

(3) Type of matrix (global, between, and within). Note that all three types of matrices are of size $n=5$, so the results can be easily compared. A slight problem is introduced by the fict that subjects generated five within matrices (one for each attribute), but only one between or global matrix. To simplify comparisons across types of matrices, in all subsequent analyses the results of the within attribute judgments are based on average values, taken across the five attributes, for each of the analysis criteria (sud as RI, SOR, etc.).

Somewhat surprisingly, we found no significant differences between the results for the three types of matrices. Sinœ this pattem holds for all the criteria considered, all subsequent figures and tables present results avenged across the three types of matrices.

Measures of goodness of fit: Table 2 presents the mean goodness of fit for each of the three solutions and scales compared. Recall that each of these indices is computed in a diffent metric and, therefore, comparisons between solutions are not possible. Note however, that for each solution method the scales are ranked identically in terms of the goodness of their implied solutions. The seven scales can be clustered into three coarse but distinct groups: At one end we observe excellent levels of fit for the two MAHP scales (7 and 8- 
based) and the "square root" scale; At the other extreme we observe extremely low levds of fit for the "squared" scale and the REMBRANDT' scale with $\tau_{l}=\ln (2)=0.7$. Saaty's linear scale and the REMBRANDT scale with $\tau_{2}=\ln \sqrt{2} \approx 0.35$ are in between (but much closer to the desirable end). Note that this clustering conesponds with the grouping of the seven scales in terms of their IQR $\left(\mathrm{s}_{\mathfrak{i}}\right)$ in Table 1.

TABLE 2. Mean (and SD) of goodness of fit by response scale and scaling method

\begin{tabular}{|c|c|c|c|}
\hline Response Scale & EV & LLS & WLS \\
\hline MAHP (8-Based) & $.05 \quad(0.06)^{l}$ & $.34 \quad(0.36)$ & $.10(0.12)$ \\
\hline Power $(\alpha=0.5)$ & $.07 \quad(0.04)$ & $.43 \quad(0.24)$ & $.13 \quad(0.09)$ \\
\hline MAHP (7-Based) & $.10 \quad(0.15)$ & $.58 \quad(0.79)$ & $.16 \quad(0.22)$ \\
\hline 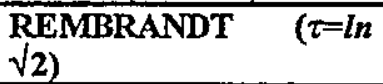 & $.28 \quad(0.23)$ & 1.55 (1.11) & $.16(0.22)$ \\
\hline Saaty & $.31 \quad(0.20)$ & $1.72 \quad(0.94)$ & $.79 \quad(1.00)$ \\
\hline REMBRANDT $(\tau=\ln 2)$ & $1.76 \quad(2.21)$ & $6.19 \quad(4.45)$ & $18.20 \quad(98.07)$ \\
\hline Power $(\alpha=2.0)$ & $1.87 \quad(1.70)$ & $6.89 \quad(3.76)$ & $16.41 \quad(54.01)$ \\
\hline Mean & $\begin{array}{ll}.63 & (1.29)\end{array}$ & $2.53 \quad(3.45)$ & $5.21 \quad(42.60)$ \\
\hline
\end{tabular}

Variance and entropy of scale values: Tables 3 and 4 present the variance and entropy of the priority weights for the 21 solutions compared. The results for these two criteria are highly similar, so they can be disaussed jointly. There are several noticeable features in both tables. With only a fw minor and insignificant exceptions, the seven scales are ordered identically for the three solutions, and the three solutions are ordered identically for each of the seven scales. Clearly, the EV and LLS solutions are practically identical and they yield slightly more homogeneous (and less informative) weights than the WLS solution. The seven scales are clustered in the same three classes identified above: The two "stretched" scales (squared and REMBRANDT scale with $\tau_{I}=\ln (2)=0.7$ are chancterized by the highest levels of diferentiation between weights, the three "shrunken" scales (the two MAHIP scales and the square root scale) yield the most homogeneous weights and, as before, the central cluster consists of the linear scale and the REMBRANDT scale with $\tau_{2}=\ln \sqrt{2} \approx 0.35$. Finally, note that the WLS solution is considerably more sensitive to the nature of the scale used than the other two.

TABLE 3. Mean (and SD) of variance of weights by response scale and scaling method

\begin{tabular}{|c|c|c|c|c|}
\hline Response Scale & EV & LLS & WLS & Mean \\
\hline MAHP (8-Based) & $.01 \quad(0.01)$ & $.01 \quad(.01)$ & $.01)^{\left(0.01^{\circ}\right.}$ & $.01(.01)$ \\
\hline Powa $(\alpha=0.5)$ & $.01 \quad(.003)$ & $.01 \quad(.003)$ & $.01)^{(.004}$ & $.01 \quad(.003)$ \\
\hline MAFP (7-Based) & $.01 \quad(0.01)$ & $.01 \quad(0.01)$ & $.01)^{(0.02}$ & $.01 \quad(0.02)$ \\
\hline Saaty & $.03 \quad(0.01)$ & $.03 \quad(0.01)$ & $.04)^{(0.02}$ & $.03 \quad(0.01)$ \\
\hline $\begin{array}{l}\text { REMBRANDT } \quad(\tau=\ln \\
\sqrt{2})\end{array}$ & $.03 \quad(0.01)$ & $.03 \quad(0.02)$ & $.04)^{(0.04}$ & $.03(0.02)$ \\
\hline REMBRANDT $(\tau=\ln 2)$ & $.07 \quad(0.02)$ & $.07 \quad(0.03)$ & $.09)^{(0.04}$ & $.08 \quad(0.04)$ \\
\hline Power $(\alpha=2.0)$ & $.07 \quad(0.03)$ & $.07 \quad(0.03)$ & $.10)^{(0.04}$ & $.08 \quad(0.03)$ \\
\hline Mean & $.03 \quad(0.03)$ & $.03 \quad(0.03)$ & $.04)^{(0.04}$ & \\
\hline
\end{tabular}


TABLE 4. Mean (and SD) entropy of weights by response seale and scaling method

\begin{tabular}{|c|c|c|c|c|}
\hline Response Scale & EV & LLS & WLS & Mean \\
\hline MAHP (8-Based) & $.09 \quad(0.07)$ & $.09 \quad(0.07)$ & $.10 \quad(0.09)$ & $.09 \quad(0.08)$ \\
\hline Powar $(\alpha=0.5)$ & $.10 \quad(0.03)$ & $.10 \quad(0.03)$ & $.11 \quad(0.04)$ & $.10 \quad(0.03)$ \\
\hline MAHP (7-Based) & $.13 \quad(0.12)$ & $.13 \quad(0.12)$ & $.15 \quad(0.17)$ & $.14 \quad(0.14)$ \\
\hline Saaty & $.32 \quad(0.10)$ & $.33 \quad(0.10)$ & $.41 \quad(0.14)$ & $.35 \quad(0.12)$ \\
\hline $\begin{array}{l}\text { REMBRANDT } \quad(\tau=\ln \\
\sqrt{2})\end{array}$ & $.32(0.14)$ & $.33(0.15)$ & $.41(0.20)$ & $.35 \quad(0.17)$ \\
\hline REMBRANDT $(\tau=\ln 2)$ & $.76 \quad(0.29)$ & $(0.29)$ & $.97 \quad(0.30)$ & $(0.31)$ \\
\hline Power $(\alpha=2.0)$ & $.75 \quad(0.22)$ & $.79 \quad(0.2)$ & $1.04(0.28)$ & $.86 \quad(0.27)$ \\
\hline Mean & $.35 \quad(0.32)$ & $.36 \quad(0.33)$ & $.45 \quad(0.42)$ & $.39 \quad(0.36)$ \\
\hline
\end{tabular}

Order reversals: In the next analysis we focus on the ordinal properties of the data and the solutions. Table 5 displays the number of strong order reversals. In evaluating these results, keep in mind that the maximal number of reversals is a finction of the matrix size In general, $0 \leq W O R$, SOR $\leq n(n-1) 2$, and in our case 0 $\leq$ WOR, SOR $\leq 10$. The most salient fature of the Table 5 is that WLS induces almost twice as many order reversais as LLS and EV for most, and across all, scales examined. Consider now the rate of order violations observed for the different scales when using EV and/or LLS solutions. In general the most accurate and fithfil representation of the original ordering of the apartments, is obtained under the family of power transformations $(3 \% \leq$ SOR $\leq 3.35 \%$ ), followed by the fimily of expcnential transformations (i.e the REMBRANDT scales, where $4.33 \% \leq$ SOR $\leq 4.84 \%$ ), and the worst results are obtained for MAHP $(5.67 \% \leq$ SOR $\leq 6.47 \%)$. We do not present a similar table of WOR because, with only two exceptions (the REMBRANDT scales aratyzed by LLS), all combinations of scales and solutions yield, pradically, identical rates of order violations (between 0.46 and 0.47 , i.e. slightly under $5 \%$ ).

TABLE 5. Mean (and SD) number of strong order reversals by response sale and scaling method

\begin{tabular}{|c|c|c|c|c|}
\hline Response Scale & $\mathbf{E V}$ & LLS & WLS & Mean \\
\hline Power $(\alpha=0.5)$ & $.30 \quad(0.61)$ & $.34 \quad(.67)$ & $.50 \quad(0.76)$ & $.38 \quad(.68)$ \\
\hline Saaty & $.30 \quad(0.61)$ & $.32 \quad(.61)$ & $.60 \quad(0.80)$ & $.40 \quad(.70)$ \\
\hline Powar $(\alpha=2.0)$ & $.43 .(0.61)$ & $.34 \quad(.67)$ & $1.03(1.07)$ & $.56 \quad(.87)$ \\
\hline REMBRANDT $(\tau=\ln 2)$ & $.57 \quad(0.65)$ & $.46 \quad(.68)$ & $97 \quad(1.07)$ & $.62 \quad(.85)$ \\
\hline MAHP (8-Based) & $.65 \div(0.76)$ & $.57 \quad(.73)$ & $78 \quad(0: 93)$ & $.64 \cdot(.81)$ \\
\hline MAHP (7-Based) & $.43 \quad(0.81)$ & $.65 \quad(.78)$ & $.82 \quad(1.00)$ & $.70 \quad(.87)$ \\
\hline REMBRANDT $(\tau=\ln \sqrt{2})$ & $.43:(0.65)$ & $.48 \quad(.64)$ & $1.20(1.24)$ & $.70 \quad(.95)$ \\
\hline Mean & $.43 \quad(0.68)$ & $.45 \quad(.69)$ & $.84 \quad(1.01)$ & $.57 \quad(.83)$ \\
\hline
\end{tabular}

Similarity of solution: We calculated the (Eudidean) distances between all 21 solutions. To examine the pattern of distances, we perfrmed a Metric Multidimensional Scaling of this matix (e.g Davison, 1983; Schiffnan, Reynolds \& Young, 1981). This analysis represents the various solitions as points in a twodimensional space. The confguration of points is displayed in Figure 3. This two dimensional representation fits the data almost perfetly (Stress $=0.02$ ). To acilitate the intepretation of the resulting confguration we added a Ew separation lines. The first (hoizontal) dimension refects the stretching of the response scale and the two vertical lines separate between the three clusters described above with the three "shrunken scales" at the leff end and the two "stretched" scales at opposite end. The second dimension reflects the method of solution used The horizontal line separates between the WLS solutions (below the line) and the EV and LLS solutions. Note that, in most cases, these two solutions for a given type of scale are "nearest neiglbors" and, for all practical purposes, indistinguishable 


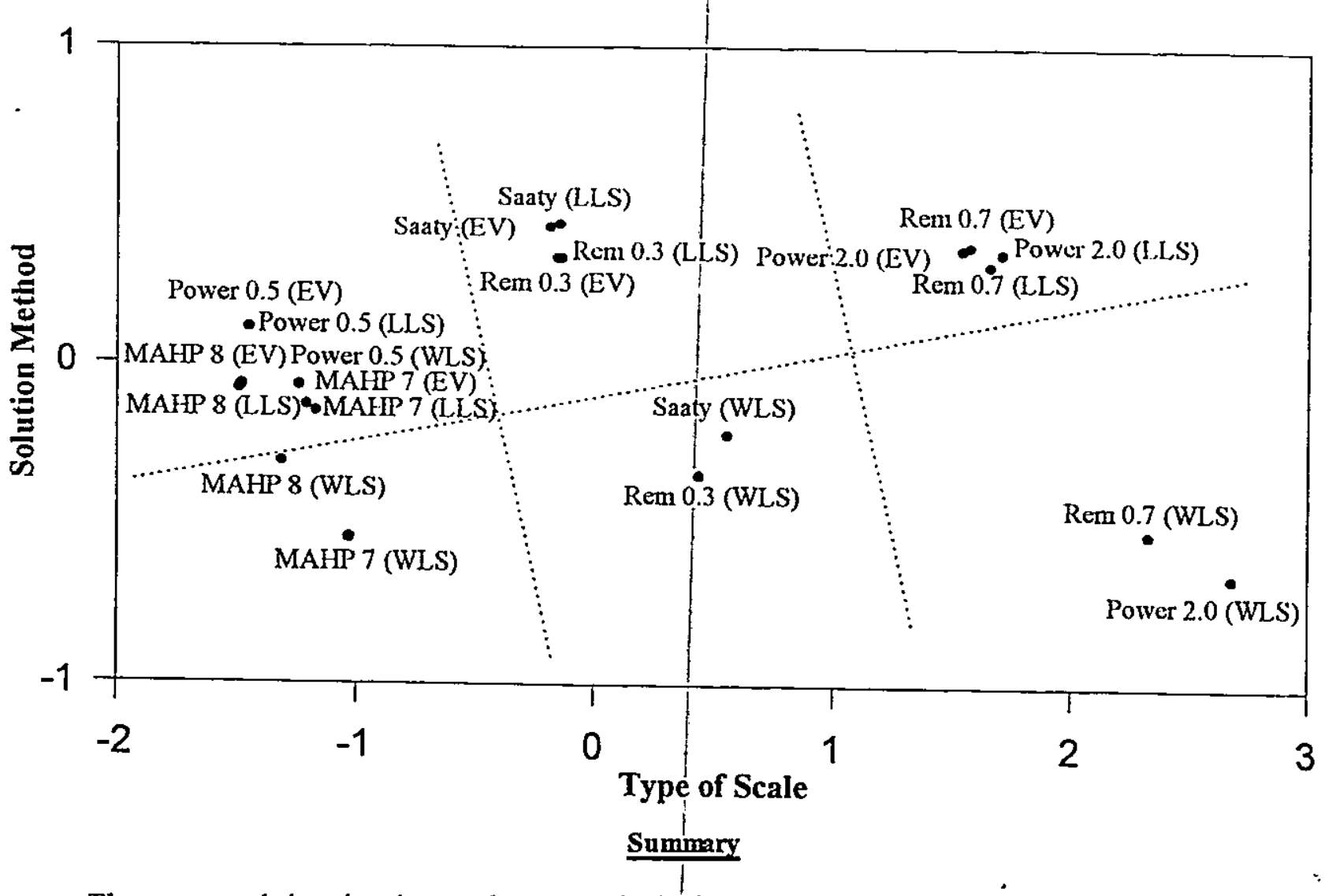

The present study is unique in several ways. It is the first systematic comparison of a large number of ninepoint scales, in conjunction with several solutions. Unlike most methodological studies in this domain, which tend to compare solutions of artificially simulated matrices, we analyzed actual judgments obtained in the course of a controlled experiment. The obvious drawback of this fict is that our results may reflect, to some degree, the specific atures of the decision problem (the number and nature of attributes and apartments presented, peculiarities of the sample, etc.). On the other hand, this methodology has allowed us, for the first time, to contrast various characteristics of solutions extracted from different types of matrices without invoking any specific assumptions. Obviously, our condusions need to be replicated and validated with a much larger, and more diverse, set of matrices. With this cautionary note in mind, we tum now to a summary of our main results.

The first, and most important, generalization from all our analyses is that the choice of scale is of crucial importance. Our results indicate that all characteristics of the solutions (goodness of fit, information number of order violations, etc.) were affeted by the nature of the scale and, in some cases, the effets were quite dramatic. Also note that the effets generalized across the three metbods of solution, so one cannot bypass the problem of choosing a scale by invoking some "scale invariant" estimation method. Furthermore, note that in all our analyses, the effets due to the choice of scale were more extreme than differences associated with the choice of the solution method.

The second condusion is that the effects of the scale choice are systematic and can be predicted, with some levd of accuracy, from the nature of the scale. Recal that in all our analyses the seven scales clustered in the same three groups which corresponded, roughly, to the degree to which the 1-9 scale was stretched/shrunken. This is most clearly illustrated in the MDS of the 21 solutions. An interesting and unexpected, observation is that this clustering is better precicted by the scales' IQR rather than their variances. To understand this pattern we re-examined the original matrices and tabulated the frequency of use of the various points on the scale. It turns out that on the average, the extreme points of the ninepoint scale are underused by comparison with those in its center. Specifically, only $9.5 \%$ of the judgments are " 1 " or " 2 " and $13.6 \%$ are " 8 " or " 9 " while the central five values $(3-7)$ represent $76.9 \%$ of the tota judgments ${ }^{3}$. Thus, it is not surpising that our results are better predicted by measures of scatter that underplay the effects of the extreme values.

${ }^{3}$ This result casts some doubts on the validity of the standard practice of assuming in simulation studies that responses are unifomly distributed across all scale values. 
Interestingly, the transformations of the scales have opposite effects on the goodness of fitconsistency of the solutions and the information/variance of the priority weights. In general, "stretching" the scale by increasing the distance between its points tends to decrease the goodness of fit but, at the same time it increases the degree of differentiaion between the priority weights (obviously, "condensing" the scale, by bringing its points closer together, has the opposite effet). In our opirion, it is important that users of the AHP be sensitive to this tradeoff Sometimes, attempts to maximize the consistency of the judgments may induce a very conservative use of the scale, i.e. using only a limited set of values and/or treaing them as more homogeneous than originally intended. Such a process may yield highly consistent, but totaly uninformative solutions. A better strategy would be to strive for solutions with maximal information, and acceptable levels of consistency (e.g a CR $<0.1$, as suggested by Saaty, 1977).

A surpising result in our analyses is the absence of systematic diffrences between the three types of matrices.

Two aspects of our study cause us to intepret this result very cautiously and reserve judgnent regarding its generalizability. We designed our study such that all judgment matrices be of the same order, and all judgments be perfmed in a relatively shot time However, in many decisions the number of comparisons is larger and, typically, the number of alternatives is larger than the number of attributes considered. We suspect that the way in which DMs use the scale is affected by the number of entities that are being compared. Our second reservation is motivated by one aspect of the analysis. Recall, that in orde to simplify comparisons between the three types of matrices we averaged the within-attibute results across all attributes. Closer inspection of the responses for the attribute-specific judgments revealed some substantial differences across attributes. For example, when subjects compared the altenatives along the most important attribute they used the high end of the scale ( 8 and 9 ) in $29.1 \%$ of the judgments and the lowe end (1 and 2) in $23.6 \%$ of the cases. By contrast, when the alternatives are compared along the least important attribute, the two ends of the scale were used in $13.6 \%$ and $38.2 \%$ of the cases, respectively. In other words, there seems to be a propensity to use extreme values ôr important attributes and to ŝvar indifierence judgments for the nonimportant ones. It is possible that by combining together all the attibutes somereal effects were obsarred. We plan to follow up this poirt with more powerfil and sensitive analyses in fiture studies.

Although our primary interest was in a comparison of the scales, we cant ignare the implications of our,results to the long controversy regading the choice.of the appropriate method of scaling. Consistent with many previous studies (e.g. Golany \& Kress, 1993); we found that in the vast majarity of cases the.EV and LLS are, practically, indistinguishable. In our opinion; they are superior to WLS in at least two important senses. : First, they are more likdy to preserve the ordinal properties of the original judgment (see also Golany \& Kress, 1993, on this point). Second, and more in line with the focts of our work, WLS was shown to be more sensitive to nature of the scale used for all the criteria considered.

\section{References}

Barzilai, J.; Cook, W., \& Golany, B. (1987). Consistent weights for judgment matrices of the relaive importance of altematives. Operations Research Letters, 6, 131-134.

Budescu, D.V. (1984). Scaling binay comparison matrices: A comment on Narsimhan's proposal. Fuzzy Sets and Systems, 14, 187-192.

Budescu, D.V., Zwick, R., \& Rapoport, A. (1986). A comparison of the eigenvalue method and the geometric mean procedure for ratio scaling. Applied Psychological Measurement 10, 69-78.

Chu, A.T.W., Kaiła, R.E., \& Spingarn, K. (1979). $\dot{A}$ comparison of two methods for detemining the weights of belonging' to fuzzy sets. Joumal of Optimization Theary and Applications, 27, 531-538.

Cogger, K.O., \& Yu, P.L. (1985). Eigenweight vectors and least distance approximation for revealed prefrence in pairwise weight ratios. Joumal of Optżizization Theory and Applications, 46, 483491.

Cook, W.D. \& Kress, M. (1992). Or̈dinal Infómation aid Preference Structures: Decision Modds and Applications. Englewood Clif, NJ: Prentice Hall

Craword, G.B. (1987). The geomëtric mean procedure for estimating the scale of a judgment matrix. Mathematical Modding, 9, 327-334.

Crawford, G., \& Williams, C. (1985). A note on the analysis of subjective judgment matrices. Joumal of Mathematical Psydology, 29, 387405.

delong, P. (1984). A statistical approach to Saaty's scaling method for priorities. Joumal of Mathematical Psychology, 28, 467-478.

Davison, M.L. (1983). Multidimeńsional Scaling. New York Wiley.

Dodd, F.J., Donegan, H.A., \& McMrster, T.B.M. (1995). Scale horizons in analytic hierarchies Joumal of Multi-Criteria Decision Anahsis, 4, 177-188. 
Donegan, H.A., Dodd, F.J., \& McMaster, T.B.(1992)

A new approach to AHP decision making. The Statisticion, 41, 295-302.

Golany, B., \& Kress, M. (1993). A multicriteria evaluation of methods for obtaining weights from ratio-scale matrices. European Joumal of Operational Research, 69, 210-220.

Gulliksen, H. (1959). Mathematical solutions forpsychological problems. American Scientist, 47, 178-201.

Gulliksen, H. (1975). Characteristic roots and vectors indicating agreement of data with different scaling laws. The Indian Joumal of Statistics, 37, 363-384.

Harker, P.T. (1987). Altemative modes of questioning in the analytic hierarchy process. Mathematical Modeling, 9, 353-360.

Jensen, R.E. (1984a). Aggregation (Comparison) schema for eigenvector scaling of criteria pricrities in hietarchical structures. Multivariate Behavioral Research, 18, 63-84.

Jensen, R.E. (1984b). An altenative scaling method for priorities in hierarchical structures. Joumal of Mathematical Psychology, 28, 317-332.

Lootsma, F.A. (1993). Scale sensitivity in the multiplicative AHP and SMART. Joumal of Multi-Criteria Decision Analysis, 2, 87-110.

Noble, E.E, \& Sanchez, P.P. (1993). A note on the information content of a consistent pairwise comparison judgment matrix of an AHP decision make. Theory and Decision, 34, 99-108.

Olson, D.L., Fliedner, G., \& Curie, K. (195). Comparison of the REMBRANDT system with anafytic hierarchy process. European Joumal of Operations Research, 82, 522-539.

Payne, J.W., Betman, J.R., \& Johnson, E.J. (1993). The Adaptive Decision Maker. Cambridge: Cambridge University Press.

Pöyhönen, M.A., Hämäläinen, R.P., \& Salo, A.A. (1996). An experiment on the numerical modeling of verbal ratio statements. Joumal of Multi-Criteria Decision Analysis, in press.

Saaty, T.L. (1977). A scaling method for pricities in hierarchical structures. Joumal of Mathematical Psychology, 15, 234-281.

Saaty, T.L. (1980). The Analytical Hierarchy Process. New York McGrawHill.

Saaty, T.L. (1986). Axiomatic fundation of the Analytic Hiemrchy process. Management Science, 32, 841855.

Saaty, T.L. (1990a). An exposition of the AHP in reply to the paper "Remarks on the Analytic Hierarchy Process", Management Science, 36, 259-268.

Saaty, T.L. (1990b). Eigenvector and logarithmic least squares. European Joumal of Operations Research, $48,156-160$.

Saaty, T.L. (1993). 'What is relative measurement? The ratio scale phantom. Mathematical Computer Modelling, 17, 1-12

Saaty, T. L. \& Keams, K P. (1985): Anahtical Plaming. New York. Pergamon Press

Saaty, T. L. \& Vargas, L. G. (1982): :The logic of p̈ricrities: Applications in business, energy, health and transportation. Boston: Kluwer NijhoffPublishing.

Saaty, T.L., \& Vargas, L.G. (1984). Comparison of eigenvalue, logarithmic least squares and least squares methods in estimating ratios. Mathematical Moddling, 5, 309-324.

Saaty, T. L. \& Vargas, L. G. (1991). Prediction, Projection and Forecasting. Boston: Kluwer Publishing.

Schiffman, S.S., Reynolds, M.L., \& Young, F.W. (1981). Introduction to multidimensional scaling: Theory methods and applications. New York Academic Press.

Takeia, E., Cogger, K.O., \& Yü, P.L. (1987). Estimating criterion weights using eigenvectors: A comperative study. European Joumal of Operations Research, 39, 360-369.

Torgerson, W.S. (1958). Theory and methods of scaling. New York. Wiley.

Webe, M., \& Borcherding K. (1993). Behavioral influences on weight judgenents in multiattribute decision making. European Joumal of Operational Research, 67, 1-12.

Zahedi, F. (1986a). The analytic hierarchy process - A survey of the method and its applications. Interfaces, 16, 96-108.

Zahedi, F. (1986b). A simulation study of estimation methods in the Anatytical Hierarchy Process. SocioEconomic Planning Sciences, 20, 347-354. 